\title{
A New Approach for Solving the Inverse Kinematics of Continuum Robot Based on Piecewise Constant Curvature Model
}

\section{Haoran Wu}

Beihang University School of Mechanical Engineering and Automation https://orcid.org/0000-00031655-4334

\section{Jingjun Yu}

Beihang University

\section{Jie Pan}

Beihang University School of Mechanical Engineering and Automation

Xu Pei ( $\nabla$ peixu@buaa.edu.cn )

Department of Mechanical Design, Beihang University

\section{Original Article}

Keywords: Continuum robot, Constant curvature model, Inverse kinematics, Geometric method

Posted Date: October 12th, 2021

DOl: https://doi.org/10.21203/rs.3.rs-952413/v1

License: (9) This work is licensed under a Creative Commons Attribution 4.0 International License. Read Full License 


\section{Title page}

\section{A New Approach for Solving the Inverse Kinematics of Continuum Robot Based on Piecewise Constant Curvature Model}

Haoran Wu, born in 1998, is currently a master candidate at Robotics Institute, Beihang University, China. His research interests include snake-arm robot and continuum robot.

E-mail: wuhaoran@buaa.edu.cn

Jingjun Yu, born in 1974, is currently a professor at Robotics Institute, Beihang University, China. He has published more than 160 papers. His research interests include compliant mechanisms and precision machine design.

E-mail: jjyu@buaa.edu.cn

Jie Pan, born in 1991, is currently a PhD candidate at Robotics Institute, Beihang University, China. His research interests include continuum robot and smart materials.

E-mail: panjie@buaa.edu.cn

Xu Pei, born in 1979, is currently an associate professor at Department of Mechanical Design, Beihang University, China. His research interests include mechanism and compliant mechanisms.

E-mail: peixu@buaa.edu.cn

Corresponding author: Xu PEI E-mail: peixu@buaa.edu.cn 


\title{
A New Approach for Solving the Inverse Kinematics of Continuum Robot Based on Piecewise Constant Curvature Model
}

\author{
Haoran $\mathrm{Wu}^{1} \bullet$ Jingjun $\mathrm{Yu}^{1} \bullet \mathrm{Jie} \mathrm{Pan}^{1} \bullet \mathrm{Xu} \mathrm{Pei}^{2 *}$
}

\begin{abstract}
The inverse kinematics of continuum robot is an important factor to guarantee the motion accuracy. How to construct a concise inverse kinematics model is very essential for the motion control of continuum robot. In this paper, a new method for solving the inverse kinematics of continuum robot is proposed based on the geometric and numerical method. Assumed that the deformation of the continuum robot is Piecewise Constant Curvature model (PCC), the envelope surface of the continuum robot based on single-segment is modeled and calculated. The clustering method is used to calculate the intersection of the curves. Then, a distinct sequence is designed for solving the inverse kinematics of continuum robot, and it is also suitable for the multi-segment continuum robots in space. Finally, the accuracy of the inverse kinematics algorithm is verified by the simulation and numerical experiment. The experiment results illustrate that this algorithm is with higher accuracy compared with the Jacobian iterative algorithm.
\end{abstract}

Keywords: Continuum robot $\bullet$ Constant curvature model • Inverse kinematics $\bullet$ Geometric method

\section{Introduction $^{12}$}

Continuum robot is a new type of bionic robot with a continuous backbone without joints [1]. Compared with traditional rigid discrete robots, it can change its shape flexibly according to environmental obstacles and has strong adaptability to small environments and unstructured spaces [2,3]. Continuum robots have been used in exploration [4], medicine [5], rescue [6] and other fields [79]. As a kind of hyper redundant robot, modeling and solving the inverse kinematics of continuum robots proves to be a very interesting and challenging problem. A large proportion of efforts in the area have focused on the

$\triangle$ Xu PEI

peixu@buaa.edu.cn

1 Robotics Institute, Beihang University, Beijing, 100191, China

2 Department of Mechanical Design, Beihang University, Beijing 100191, China establishment and solution of kinematic model.

Geometric [10] and mechanical [11] methods are widely applied in the modeling of continuum robots. Chen [12] used quadratic Bezier curve to establish the continuum robot model and sensor data is used to compensate error. Chirikjian [13] and Agrawal [14] link the shape of a particular mathematical curve to a high-degree-of-freedom (DOF) robot. Another approach is choosing an arc which closely matches the kinematics of continuum robots, due to the equal distribution of forces inherent in the design of continuum robot [5]. And the Piecewise Constant Curvature (PCC) model is also carried out by Bryan A. Jones [15] under this assumption, an assumption also made throughout this paper. Closed position and velocity kinematics [16] are also established on account of PCC model. Based on the PCC model, Zheng Li [17] used screw to build continuum robot kinematics model and solve its workspace. Tobias Mahl [18] and Sears [19] constructed Variable Constant Curvature (VCC) model for continuum robot with variable curvature, which divides each joint into multiple segments.

The traditional method of solving the inverse kinematics of continuum robot is the generalized inverse method $[20,21]$. The continuum robot is transformed into rigid body model and solved by iteration of Jacobian inverse matrix. But non-convergence and singularities are also inevitable through this method. Neural network [22-24] is also used to solve the inverse solution of continuum robot, which is also widely used for control and modeling of continuum robots. However, excessive calculation may be existed due to the large training set when the robot has many degrees of freedom. Another method is to establish the model according to the geometric relationship, and the inverse kinematics can be solved by numerical iteration, which is also applied in this paper. Williams [25] and Svenja [26] analogized the movement of a snake's tail following the head's trajectory, and proposed a follow theleader (FTL) heuristic algorithm for hyper redundant robot. Sinivas [27] proposed a geometric algorithm to get closedform inverse kinematics of multi-continuum robot. Based on PCC model, Underwater soft manipulator designed by Zheyuan Gong [28] which applied the reverse constant 
curvature model to get the inverse kinematics. However, few types of robots are suitable for this algorithm due to the limitation of the algorithm.

Combined with geometrical and numerical, a new approach for solving the inverse kinematics of continuum robot is proposed in this paper. Based on the PCC model, geometrical method is used to build the model of continuum robot in Section 2. Furthermore, a distinct algorithm is proposed in Section 3 through geometrical and numerical. The algorithm has a wide range of applicability, which is fitted for multi-segment continuum robots and continuum robots in space. Finally, the effectiveness and accuracy of the algorithm are verified by numerical experiments and simulation in Section 4.

\section{Establish Continuum Robot Kinematics Model}

\subsection{Model assumptions of continuum robot}

For the sake of analysis, the compliant deformation of a continuum robot can be decomposed into a constitution of many linkage motions. In order to emerge the movement adaptability of continuum robot veritably, spherical hinge is used to construct the model instead of Hooke, rolling pairs or twin-pivot joint [29,30]. A schematic diagram of twosegment continuum robot is shown in Figure 1 (a). Based on Piecewise Constant Curvature model (PCC), regardless of the shape of the robot's own joints and the area of the cross-section, the kinematics configuration of continuum robot can be described by the geometry of a finite arc.

The relationship between configuration parameters of continuum robot and single joint is illustrated in Figure 1 (b). The length of a single segment of continuum robot with $\mathrm{n}$ joint is 1 . Based on the PCC model, the bending angle is $\alpha$, and the direction angle is $\theta$. It is assumed that the rotation angle of each joint is identical, the specific relationship of configuration parameters is shown in Eq. (1). When the number of joints of a single segment continuum robot is 8 and the bending angle range is less than $\pi / 2$, the relative error of the length of the single segment continuum robot is less than $0.3 \%$. Therefore, for the purpose of simplify the model, it is considered that the length of a single segment continuum robot remains unchanged.

$$
\left\{\begin{array}{l}
\alpha=n \cdot \alpha_{\text {single }} \\
\theta=n \cdot \theta_{\text {single }} \\
l^{\prime}=l \cdot \tan \left(\frac{\alpha}{2 n}\right) / \frac{\alpha}{2 n} \approx l
\end{array}\right.
$$

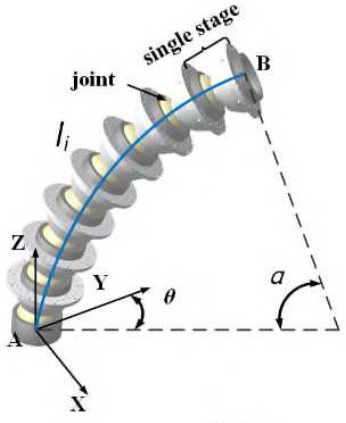

(a)

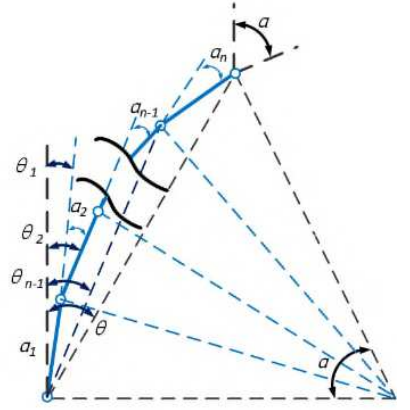

(b)
Figure 1 Single segment of Tendon-driven continuum robot

To sum up, in order to further establish the model of continuum robot, the following assumptions are proposed based on PCC model.

(1) The length of the single-stage continuum robot is constant.

(2) Continuum robot is tangent continuous and no sudden change of curvature.

(3) Continuum robot has no distortion on the $\mathrm{Z}$ axis.

(4) The influence of external loads on the shape of continuum robot is not considered.

\subsection{Envelope line/surface of single-segment continuum robot}

A schematic diagram of a single-segment continuum robot is shown in Figure 2. According to the geometric continuity, the parameter equations of continuum robot can be derived as Eq. (2) and Eq. (3).

$$
\begin{gathered}
\left\{\begin{array}{l}
x=\frac{l}{\alpha} \cdot(1-\cos \alpha) \\
y=\frac{l}{\alpha} \cdot \sin \alpha
\end{array}\right. \\
\left\{\begin{array}{l}
x=\sin \theta(1-\cos \alpha) \frac{l}{\alpha} \\
y=\cos \theta(1-\cos \alpha) \frac{l}{\alpha} \\
z=\sin (\alpha) \frac{l}{\alpha}
\end{array}\right.
\end{gathered}
$$

According to the geometric relationship, the envelope line/ surface of continuum robot can be drawn. If the starting point of the robot is known to be at point A, the tip point B must be on the envelope/surface shown in Figure 2 below, and vice versa. By solving the intersection of two or more envelopes, inverse kinematics of continuum robot can be further obtained. 
(a)

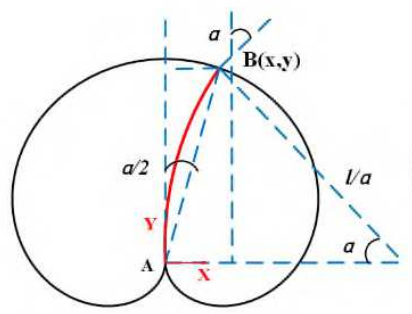

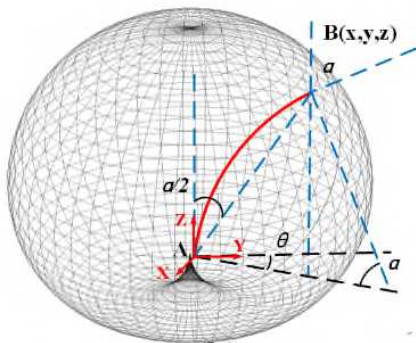

(b)
Figure 2 End envelope line/surface of single-segment continuum robot

\subsection{Tip pose of continuum robot}

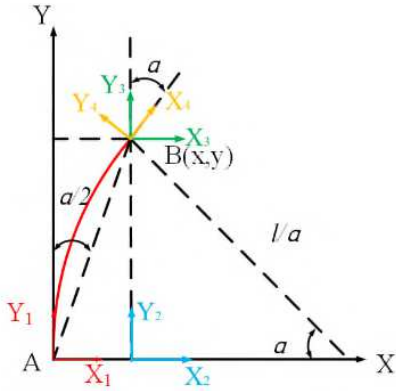

(a)

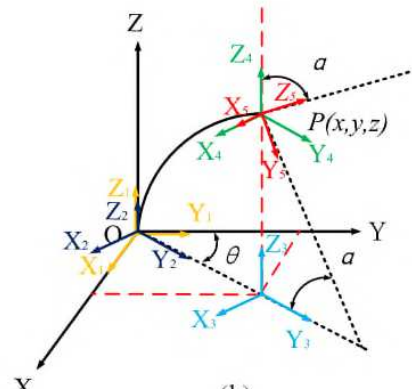

(b)
Figure 3 Coordinate transformation of continuum robot

The coordinate transformation of continuum robot in space and on the plane is illustrated in Figure 3. Through coordinate transformation, the tip pose of single-segment continuum robot is shown in Eq. (4).

$$
\begin{aligned}
T_{1} & =R_{z}\left(-\theta_{1}\right) \cdot T_{y}\left(l_{1} / \alpha_{1}\left(1-\cos \alpha_{1}\right)\right) \cdot T_{z}\left(l_{1} / \alpha_{1} \sin \alpha_{1}\right) \cdot R_{x}\left(-\theta_{1}\right) \\
& =\left[\begin{array}{cccc}
\cos \theta_{1} & \cos \alpha_{1} \sin \theta_{1} & \sin \alpha_{1} \sin \theta_{1} & \frac{-\left(l_{1} \sin \theta_{1}\right)\left(\cos \alpha_{1}-1\right)}{\alpha_{1}} \\
-\sin \theta_{1} & \cos \alpha_{1} \cos \theta_{1} & \sin \alpha_{1} \cos \theta_{1} & \frac{-\left(l_{1} \cos \theta_{1}\right)\left(\cos \alpha_{1}-1\right)}{\alpha_{1}} \\
0 & -\sin \alpha_{1} & \cos \alpha_{1} & \frac{l_{1} \sin \alpha_{1}}{\alpha_{1}} \\
0 & 0 & 0 & 1
\end{array}\right] \text { (4) }
\end{aligned}
$$

The tip pose of the two-segment continuum robot is illustrated in Appendix C.

Likewise the tip pose of continuum robot on the plane can be obtained in Eq. (5) and Eq. (6)

$$
T_{1}=T_{x}\left(l_{1} / \alpha_{1}\left(1-\cos \alpha_{1}\right)\right) \cdot T_{y}\left(l_{1} / \alpha_{1} \cos \alpha_{1}\right) \cdot R_{x}(-\alpha)
$$$$
=\left[\begin{array}{ccc}
\cos \alpha_{1} & -\sin \alpha_{1} & \frac{l_{1}}{\alpha_{1}} \sin \alpha_{1} \\
\sin \alpha_{1} & \cos \alpha_{1} & \frac{l_{1}}{\alpha_{1}}\left(1-\cos \alpha_{1}\right) \\
0 & 0 & 1
\end{array}\right]
$$

$$
T=T_{1} \cdot T_{2}=\left[\begin{array}{ccc}
\cos \left(\alpha_{1}+\alpha_{2}\right) & -\sin \left(\alpha_{1}+\alpha_{2}\right) & \frac{l_{1}}{\alpha_{1}} \cdot \sin \left(\alpha_{1}\right)+\frac{l_{2}}{\alpha_{2}} \cdot\left(\sin \left(\alpha_{1}+\alpha_{2}\right)-1\right) \\
\sin \left(\alpha_{1}+\alpha_{2}\right) & \cos \left(\alpha_{1}+\alpha_{2}\right) & \frac{l_{1}}{\alpha_{1}} \cdot\left(1-\cos \left(\alpha_{1}\right)\right)+\frac{l_{2}}{\alpha_{2}} \cdot\left(-\cos \left(\alpha_{1}+\alpha_{2}\right)+1\right) \\
0 & 0 & 1
\end{array}\right]
$$

\section{Inverse Kinematics of Continuum Robot}

\subsection{Inverse Kinematics of Continuum Robot on the Plane}

\subsubsection{Inverse kinematics of two-segment continuum robot} on the plane

As shown in Figure 4, the bending angle of the twosegment continuum robot in the plane are $\alpha_{1}$ and $\alpha_{2}$, respectively. The terminal coordinates of the continuum robot are $\mathrm{B}(\mathrm{x}, \mathrm{y})$. The general steps for solving the inverse kinematics of continuum robot are illustrated as follows.

(1) Solve the rotation angle range of the second curve

In order to calculate the tip rotation angle range $\theta$, find the two extreme positions where the two curves are tangent.

(2) Find the intersection of two curves

In the current pose, draw the first curve $S_{\boldsymbol{I}}$ and the second curve $\boldsymbol{S}_{2}$ of continuum robot. In the specific algorithm, the two curves are discretized into points. As illustrated in Appendix A, the clustering algorithm is used to solve the intersection of two curves.

If the intersection is $\left(\boldsymbol{x}_{\boldsymbol{1}}, \boldsymbol{y}_{\boldsymbol{1}}\right)$, Eq. (8) can be obtained by substituting Eq. (2) of the first continuum robot.

$$
\left\{\begin{array}{c}
\frac{l_{1}}{\alpha_{1}} \cdot \sin \alpha_{1}+\frac{l_{2}}{\alpha_{2}} \cdot\left(\sin \left(\alpha_{1}+\alpha_{2}\right)-1\right)=x_{0} \\
\frac{l_{1}}{\alpha_{1}} \cdot\left(1-\cos \alpha_{1}\right)+\frac{l_{2}}{\alpha_{2}} \cdot\left(-\cos \left(\alpha_{1}+\alpha_{2}\right)+1\right)=y_{0}
\end{array}\right.
$$

$$
\left\{\begin{array}{c}
\alpha_{1}=2 \arctan \left(\frac{2 x_{1}}{y_{1}}\right) \\
\alpha_{2}=\arcsin (T(2,1))-\alpha_{1}
\end{array}\right.
$$

(3) Make auxiliary curves at the intersection

As shown in Figure 4 (a), an auxiliary curve $\boldsymbol{S}_{\boldsymbol{1 - 2}}$ is drawn at the intersection along the tangent direction of first 
segment continuum robot. If there is a feasible solution, the tip point must pass the second curve $\boldsymbol{S}_{2}$ and the auxiliary curve $S_{1-2}$ at the same time.

(4) Find a feasible solution and check the continuity of the solution

As illustrated in Figure 4 (a), repeat step (2) and (3), a series of curves are drawn within the rotation angle range of the second curve. Then check the continuity of the solution until a feasible solution is found. A feasible solution of continuum robot is presented in Figure 4 (b).
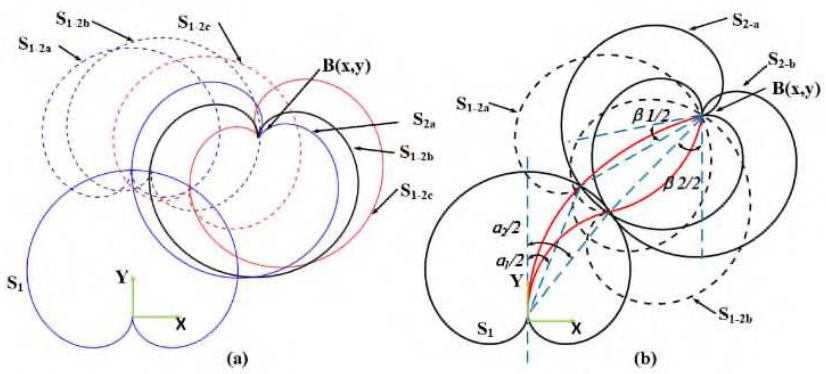

Figure 4 Inverse kinematics of two-segment continuum robot

\subsubsection{Inverse kinematics of multi-segment continuum robot on the plane}

(1) Three-segment continuum robot, giving the tip pose

Similar to the method of solving two-segment continuum robot, the rotation angle range of the second curve can be obtained. Within the rotation angle range, a series of curves are made to solve the intersection of curves and auxiliary curves are produced at the intersection as illustrated by Figure 5 (b). A feasible solution is found as shown in Figure 5 (c).

(2) Multi-segment continuum robot, giving certain constraints

There may be many inverse kinematics for multisegment continuum robots, which need to be solved under certain restricted conditions. Thus, this article will not discuss them.

\subsection{Inverse Kinematics of Continuum Robot in Space}

\subsubsection{Inverse kinematics of two-segment continuum robot in} space

As shown in Figure 6, the bending angle of the twosegment continuum robot in space are $\boldsymbol{\alpha}_{1}$ and $\boldsymbol{\alpha}_{2}$ respectively and the direction angle are $\boldsymbol{\theta}_{1}$ and $\boldsymbol{\theta}_{2}$ respectively. The terminal coordinates of the continuum robot are $\mathrm{B}(\mathrm{x}, \mathrm{y}$, $\mathrm{z}$ ). The general steps for solving the inverse kinematics of continuum robot are illustrated as follows.

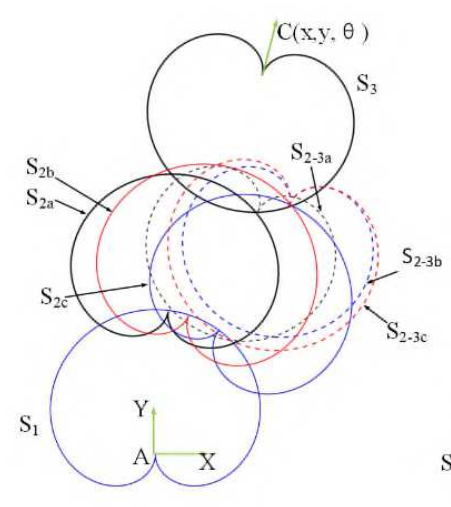

(a)

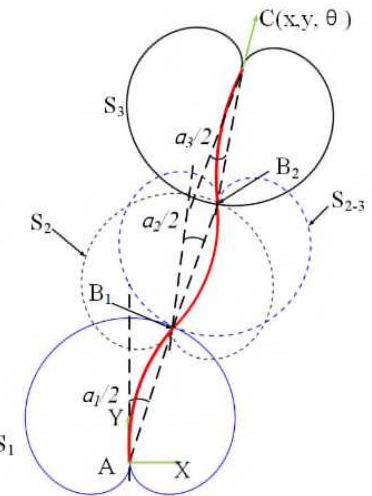

(b)
Figure 5 Inverse kinematics of multi-segment continuum robot

(1) Find the intersection of two curves

The first curve $\boldsymbol{V}_{\boldsymbol{l}}$ is generated at the origin and the second curve $\boldsymbol{V}_{2}$ is made at point $\mathrm{C}$ along the tip pose as illustrated in Figure 6. In the specific algorithm, the two curves are discretized into points, and a series of planes parallel to the OXY plane are used to intercept the two curves. Points less than a certain threshold from the intercept plane are projected onto the plane. The problem of solving the intersection of surfaces can be transformed into a problem of solving the intersection of curves on plane. And more details can be found in Appendix C.

(2) Make auxiliary curves at line of intersection

In the current tangent plane, an auxiliary curve $\boldsymbol{V}_{\boldsymbol{I - 2}}$ is made at the intersection. As shown in Figure 6 (a), if there is a feasible solution at this position, the tip point must be on both the curve $\boldsymbol{V}_{2}$ and the curve $\boldsymbol{V}_{\boldsymbol{1 - 2}}$. As illustrated in Figure 6 (b), the auxiliary curve is not at the tip point, so there is no feasible solution.

(3) Solve feasible solutions

If there is a feasible solution, and the intersection point is $\left(x_{1}, y_{1}, z_{1}\right)$. Substitute the coordinates of the intersection obtained into Eq. (6).

$$
\left\{\begin{array}{c}
\theta_{1}=\arctan \left(y_{0} / x_{0}\right) \\
\alpha_{1}=2 \arctan \left(\sqrt{x_{1}^{2}+y_{1}^{2}} / z_{1}\right)
\end{array}\right.
$$

Substitute $\boldsymbol{\alpha}_{\boldsymbol{I}}$ and $\boldsymbol{\theta}_{\boldsymbol{1}}$ into Eq. (6) , Eq. (10) can be obtained as follows.

$$
\left\{\begin{array}{l}
\sin \alpha_{1} \sin \theta_{2}=T(3,1) \\
-\cos \alpha_{1} \cos \alpha_{2} \cos \theta_{2}-\cos \alpha_{1} \cos \alpha_{2}=T(3,2) \\
\cos \alpha_{1} \cos \alpha_{2}-\cos \theta_{2} \sin \alpha_{1} \sin \alpha_{2}=T(3,3)
\end{array}\right.
$$

It can be solved by combining the above Eq. (10). 


$$
\left\{\begin{array}{l}
\theta_{2}=\arcsin \left(\frac{T(3,1)}{\sin \alpha_{1}}\right) \\
\alpha_{2}=\arcsin \left(\frac{\cos \alpha_{1} \cdot T(3,2)+\sin \theta_{2} \sin \alpha_{1} T(3,3)}{-\cos ^{2} \alpha_{1}-\cos ^{2} \theta_{2} \sin ^{2} \alpha_{1}}\right)
\end{array}\right.
$$

\section{(4) Further solve the exact solution}

On the basis of the solution obtained, the sampling rate is increased, and the angle range of the curve is determined and reduced. Repeat the above steps until a required accuracy is achieved.

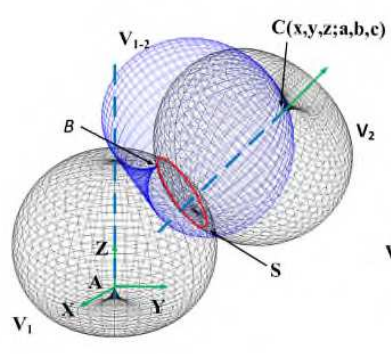

(a)

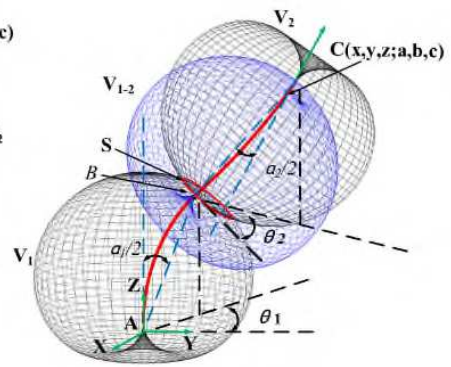

(b)
Figure 6 Inverse kinematics of two-segment continuum robot in space

\subsubsection{Inverse kinematics of multi-segment continuum robot in space}

The inverse kinematics of a three-segment continuum robot in space is illustrated in Figure 7. The position and tip pose of the continuum robot and the bending angle of the first segment of the continuum robot are known. As shown in Figure 7 (a), the second curve is made on the intersection $S_{I}$, and the third curve is made according to the tip pose. Using the same method above, the intersection $S_{2}$ of the two curves is solved. Take a point on the intersection $S_{2}$ as the auxiliary curve $S_{2-3}$. Repeat the above steps until the feasible solution is found. A feasible solution of continuum robot in this pose is shown in Figure 7 (b).

\subsubsection{General steps for solving the inverse kinematics of continuum robot}

The distinct sequence for solving the inverse kinematics of continuum robot is illustrated in Figure 8. The approximate range of the solution can be solved by using the above method. Increase the sampling rate and narrow the angle interval until a more accurate solution is obtained

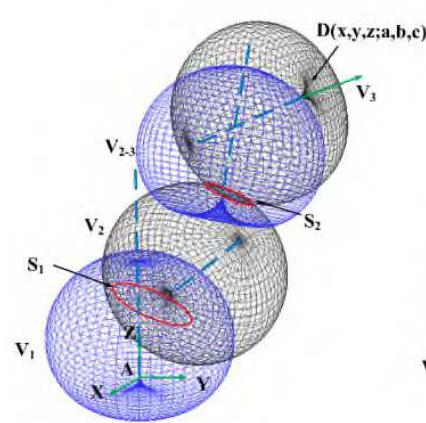

(a)

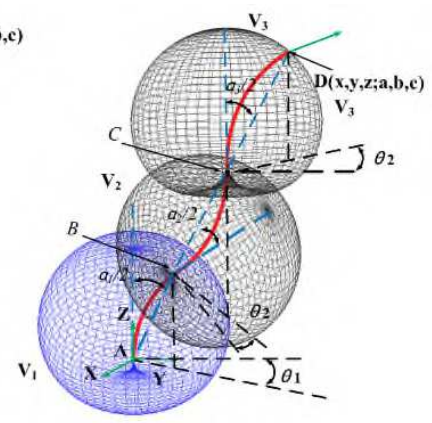

(b)
Figure 7 Inverse kinematics of three-segment continuum robot in space

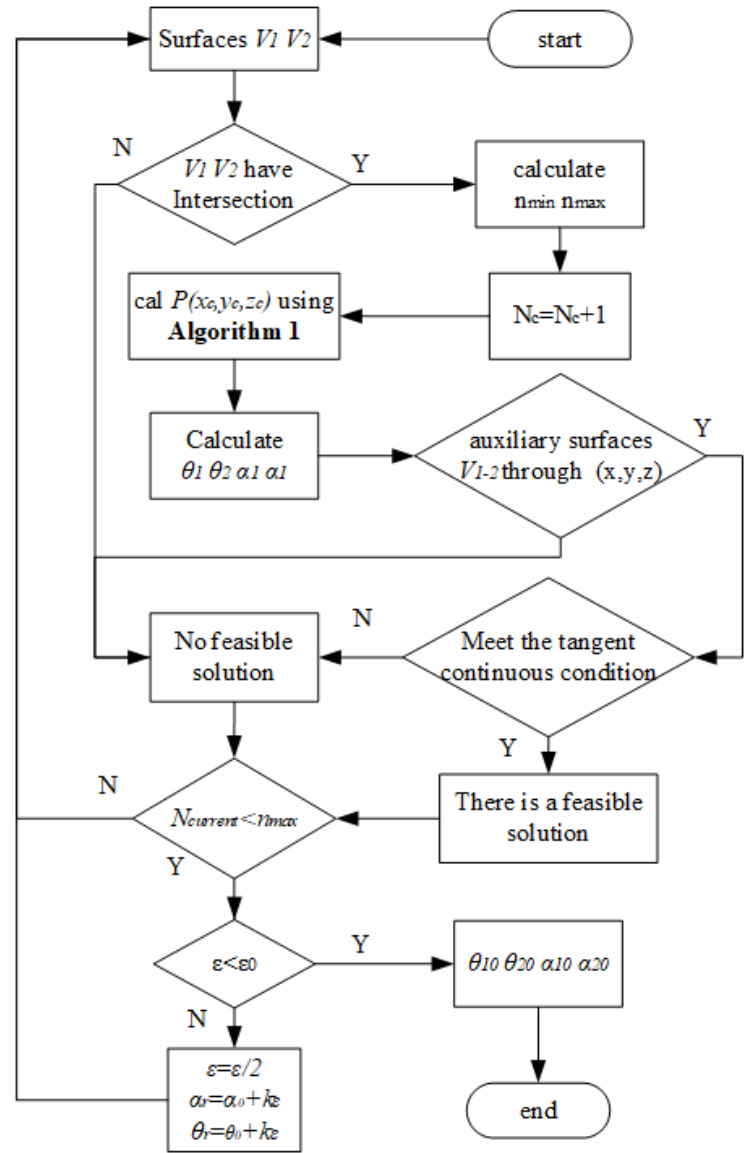

Figure 8 General steps for solving the inverse kinematics of continuum robot

\section{Comparison and Simulation Verification of Inverse Kinematics Algorithms}

\subsection{Comparison of graphical method and Jacobian method}

In order to verify the accuracy of the algorithm, the Jacobian iterative method is used for comparison [20,21]. 
The iterative relationship of this method is shown in Eq. (12).

$$
q_{i+1}=q_{i}+\xi_{i} \cdot J^{T}\left(q_{i}\right) J\left(q_{i}\right) J^{T}\left(q_{i}\right)^{-1}\left(x_{f}-k\left(q_{i}\right)\right)
$$

In Eq. (12), $q_{i}$ is the configuration parameter of robotic arm in $i$ - th iteration process. $x_{f}$ is the target position that the robot arm needs to reach, $k\left(q_{i}\right)$ is the forward kinematics of robot arm and $\rho$ is the step length of each iteration. $J^{\#}(q)$ is the pseudo-inverse of the Jacobian matrix.

$$
J^{\#}\left(q_{i}\right)=J^{T}\left(q_{i}\right) J\left(q_{i}\right) J^{T}\left(q_{i}\right)^{-1}
$$

As shown in Figure 9 (a), the single-segment continuum robot is transformed into a rigid manipulator model [15][17]. The relationship between the configuration parameters of the rigid manipulator arms the singlesegment continuum robot is illustrated in Eq. (14).

$$
\left\{\begin{array}{l}
\theta_{1}=\arctan \left(\frac{\cos (\alpha / 2)}{-\sin (\alpha / 2) \cos \theta}\right) \\
\theta_{2}=\arcsin (\sin (\alpha / 2) \sin \theta) \\
d_{3}=2 \cdot l / \alpha \cdot \sin (\alpha / 2) \\
\theta_{4}=\theta_{2} \\
\theta_{5}=\theta_{1}+\pi
\end{array}\right.
$$

As shown in Figure 9 (b), MATLAB Robotics Toolbox (RTB9.10, Petercorke, AUS) \& (MATLAB, R2019b, MathWorks.Inc, US) is used to build the robot model and solve the inverse kinematics. Two segment continuum robot is used to carry out simulation, and the length of each segment is 10 units. 500 sets of tip points in the space are randomly selects in this article, the Jacobian iteration method and the geometric method in this paper are used to solve the inverse kinematics of continuum robot. And the error distribution of Jacobian method and geometric method are illustrated in Figure 10 and Figure 11 respectively. By fitting the error data, it can be found that the error of the inverse solution approximately obeys the $\gamma$ distribution.

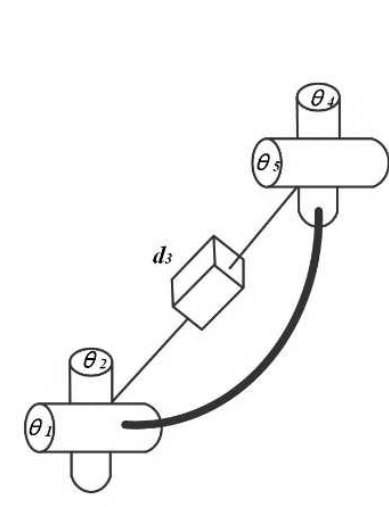

(a)

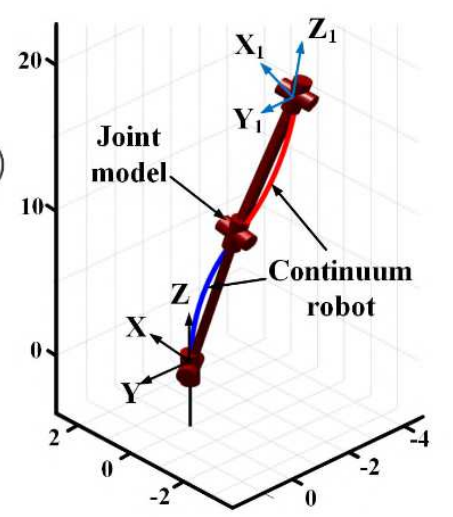

(b)
Figure 9 Solving inverse kinematics of continuum robot with MATLAB Robotics toolbox
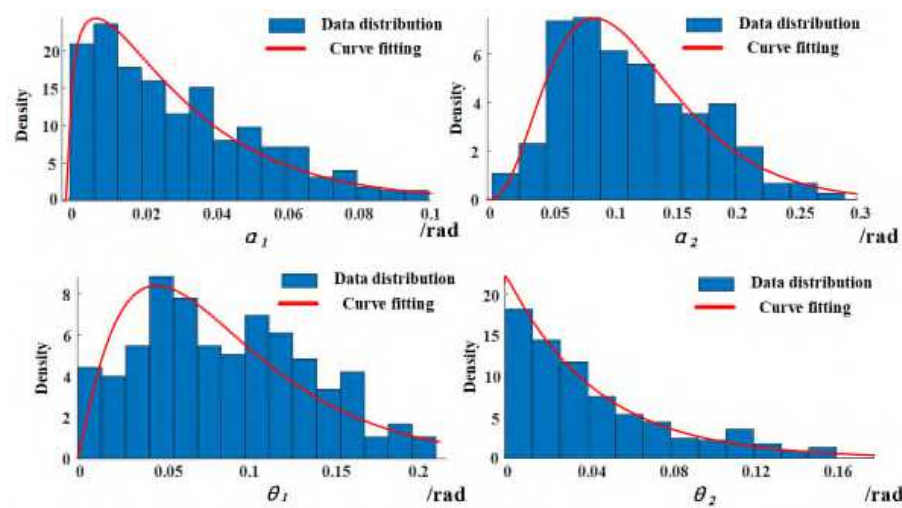

Figure 10 Error distribution of the inverse kinematics of continuum robot with Jacobian method
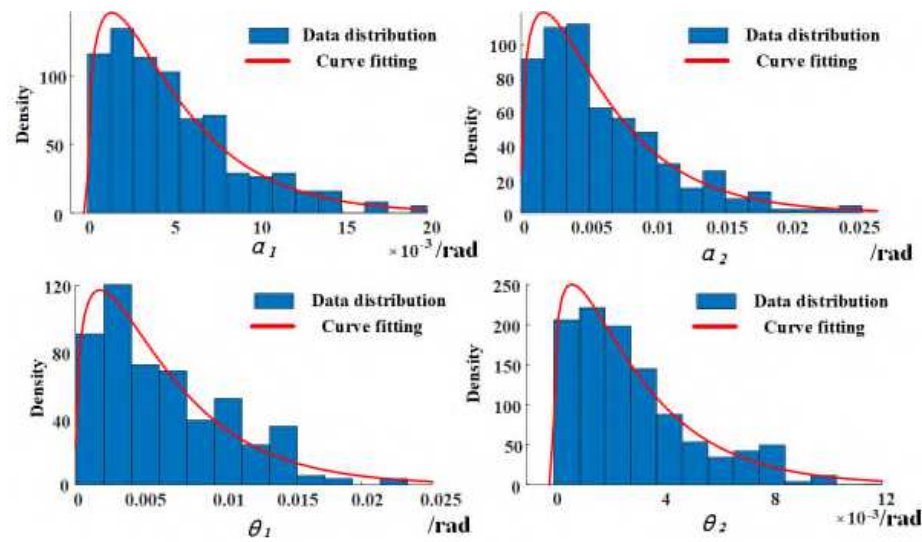

Figure 11 Error distribution of the inverse kinematics of continuum robot with graphical method

The average error of the two methods is shown in Table 1. Comparing the solution results of the two methods, the geometric method has higher accuracy. Singular poses may exist when Jacobi pseudo-inverse method is used to solve inverse kinematics. So, a small part of the inverse solution 
may have relatively large errors.

Table 1 Comparison of the error average of the two methods

\begin{tabular}{cccccc}
\hline & $\boldsymbol{\alpha}_{1} /\left(^{\circ}\right)$ & $\boldsymbol{\vartheta}_{1} /\left(^{\circ}\right)$ & $\boldsymbol{\alpha}_{2} /\left(^{\circ}\right)$ & $\boldsymbol{\vartheta}_{2} /\left(^{\circ}\right)$ & terminal error \\
\hline Jacobian & 1.72 & 5.09 & 6.87 & 2.46 & 0.17 \\
Graphical & 0.28 & 0.35 & 3.5 & 0.167 & 0.073 \\
\hline
\end{tabular}

\subsection{Simulation verification of the algorithm}

To further verify the accuracy of the algorithm, the model of the continuum robot is imported into ADAMS (ADAMS, 2017, MSC Inc., US) for kinematic simulation. Based on PCC model, the continuum robot can be divided into two segments. As shown in Figure 11, continuum robot moves from pose 1 to pose 2. Actuators are added at the joints of the model to measure the robot's terminal posture. Fifth-order polynomial curve is used to change the configuration of the continuum robot. In order to solve the inverse kinematics of continuum robot, the geometric method is used according to the tip pose of the robot.

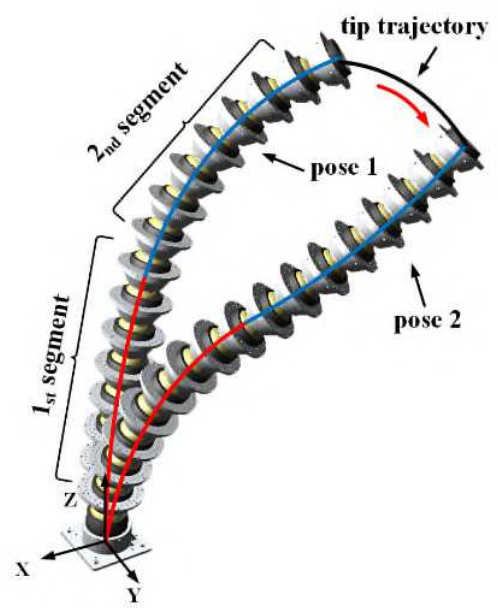

Figure 12 Simulation of continuum robot based on ADAMS

The simulation and calculated values of the configuration parameters during the robot movement are shown in Figure 12. And the absolute error of configuration parameters is illustrated in Figure 13. In addition to the relatively large errors that may exist in individual special positions, the simulated results agree well with the calculated results.

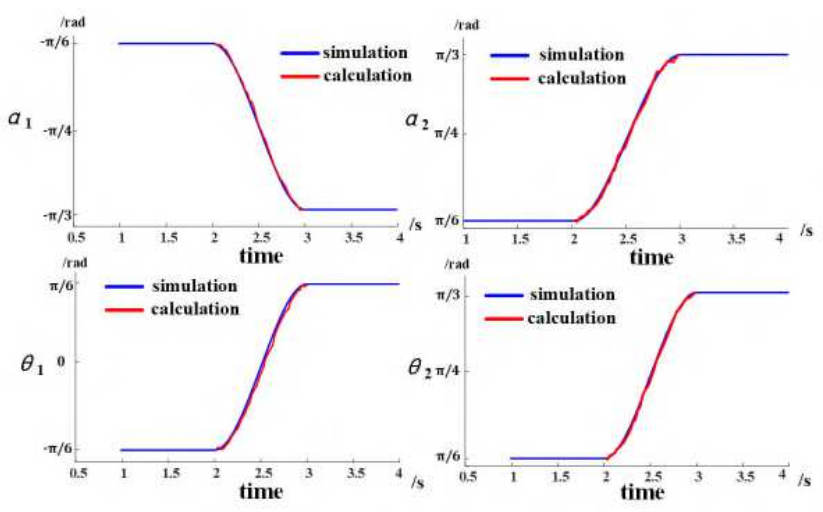

Figure 13 The simulation and calculated values of configuration parameters

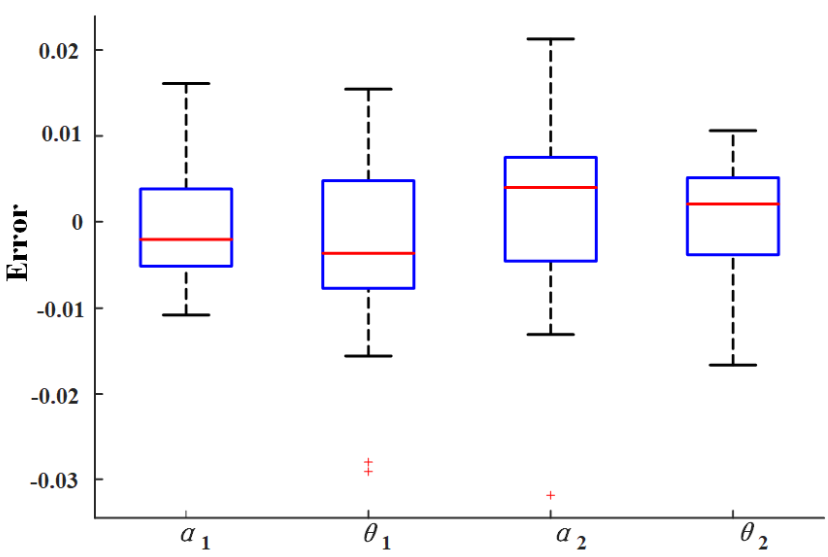

Figure 14 Absolute error of configuration parameters

\section{Discussion}

Our algorithm successfully settles the problem of inverse kinematics for multi-segment continuum robot. Due to the definite geometric meaning of the algorithm, it is convenient to understand and use. However, there are still several features and limited needed to be noted:

(1) Since K-means clustering method is used to solve the intersection of two curves/surfaces, and the initial location of the cluster center is randomly generated based on the given data. Therefore, within the allowable error range, subtle difference may be existed in each calculation.

(2) In essence, the algorithm is a numerical algorithm combining numbers and shapes by discretizing surfaces or curves into points. Therefore, the accuracy of the algorithm largely lies in the sampling accuracy of curves and surfaces. In consequence, the selection of the sampling rate needs to be determined according to the actual calculation accuracy requirements and computational efficiency. 
(3) Faced with the problem of solving the inverse kinematics of multi-segment continuum robot, large amount of calculation may be required by applying this algorithm to obtain an accurate solution. However, the approximate value can still be obtained by using this method, and it can be combined with other heuristic algorithms to obtain more accurate results.

\section{Conclusion}

A new method for solving the inverse kinematics of continuum robot is proposed in this paper. Based on Piecewise Constant Curvature model (PCC), the envelope surface and tip pose of the continuum robot is modeled and calculated. The clustering and iteration method is used to calculate the intersection of the curves. Combined with geometric and numerical method, a distinct sequence is proposed in this paper, which is well fitted for two segments continuum robots in space. Finally, the accuracy of the algorithm is verified through simulation and numerical experiment. Compared with Jacobi pseudoinverse algorithm, this algorithm has higher precision and no non-convergent or singular solution. As this algorithm is capable of definite geometric meaning, so it is easy to be accepted and applied. Therefore, the graphical method provides a new scheme for the motion control of two-stage and even multi-stage continuous robots.

\section{Declaration}

\section{Acknowledgements}

The authors sincerely thanks to Professor Pei and Doctor Pan of Beihang University for critical discussion and reading during manuscript preparation.

\section{Funding}

This work was supported in part by the National Natural Science Foundation of China (Grant No. U1813221) and the National Key Research and Development Program of China (Grant No. 2019YFB1311200)

\section{Availability of data and materials}

The datasets supporting the conclusions of this article are included within the article.

\section{Authors' contributions}

The author' contributions are as follows: Jin-Jun Yu and Xu Pei was in charge of the whole trial; Hao-Ran $\mathrm{Wu}$ wrote the manuscript; Xu Pei assist in revising manuscripts. Jie Pan assisted with Simulation analysis

\section{Competing interests}

The authors declare no competing financial interests.

\section{Consent for publication}

Not applicable

Ethics approval and consent to participate

Not applicable

\section{References}

[1] Pritts M B, Rahn C D. Design of an artificial muscle continuum robot[C]. IEEE International Conference on Robotics and Automation, 2004. Proceedings. ICRA'04. 2004, 2004: 4742-4746.

[2] Robinson G, Davies J B C. Continuum robots-a state of the $\operatorname{art}[\mathrm{C}]$. Proceedings 1999 IEEE international conference on robotics and automation (Cat. No. 99CH36288C), 1999: 2849-2854.

[3] Sun L, Hu H, Li M J R. A review on continuum robot[J], 2010, 32(5): 688-694.

[4] Gravagne I A, Rahn C D, Walker I D. Large deflection dynamics, and control for planar continuum robots[J]. Ieee-Asme Transactions on Mechatronics, 2003, 8(2): 299-307.

[5] Simaan N. Snake-like units using flexible backbones and actuation redundancy for enhanced miniaturization[C]. Proceedings of the 2005 IEEE international conference on robotics and automation, 2005: 3012-3017.

[6] Tsukagoshi H, Kitagawa A, Segawa M. Active hose: An artificial elephant's nose with maneuverability for rescue operation[C]. Proceedings 2001 ICRA. IEEE International Conference on Robotics and Automation (Cat. No. 01CH37164), 2001: 2454-2459.

[7] $\mathrm{Xu} \mathrm{K}$, Simaan N J I T O R. An investigation of the intrinsic force sensing capabilities of continuum robots[J], 2008, 24(3): 576-587.

[8] Kai X, Huan L J J O M E. Multi-backbone continuum mechanisms: Forms and applications[J], 2018, 54(13): 25-33.

[9] Qi F, Ju F, Bai D, et al. Motion modelling and error compensation of a cable - driven continuum robot for applications to minimally invasive surgery[J], 2018, 14(6): e1932.

[10] Webster R J, Iii, Jones B A. Design and Kinematic Modeling of Constant Curvature Continuum Robots: A Review[J]. International Journal of Robotics Research, 2010, 29(13): 1661-1683.

[11] Trivedi D, Lotfi A, Rahn C D J I T O R. Geometrically exact models for soft robotic manipulators[J], 2008, 24(4): 773-780.

[12] Qi F, Ju F, Bai D, et al. Motion modelling and error compensation of a cable-driven continuum robot for applications to minimally invasive surgery $[\mathrm{J}]$. International Journal of Medical Robotics and Computer Assisted Surgery, 2018, 14(6).

[13] Chirikjian G S, Burdick J W J I T O R, Automation. A modal approach to hyper-redundant manipulator kinematics[J], 1994, 10(3): 343-354.

[14] Agrawal S K, Li S, Annapragada M. Hyper-redundant planar manipulators: motion planning with discrete modal summation procedure[C]. Proceedings of the 1994 IEEE International Conference on Robotics and Automation, 1994: 1581-1586.

[15] Jones B A, Walker I D. Kinematics for multisection continuum robots[J]. Ieee Transactions on Robotics, 2006, 22(1): 43-55.

[16] Hannan M W, Walker I D J J O R S. Kinematics and the implementation of an elephant's trunk manipulator and other continuum style robots[J], 2003, 20(2): 45-63. 
[17] Li Z, Wu L, Ren H, et al. Kinematic comparison of surgical tendondriven manipulators and concentric tube manipulators[J], 2017, 107: 148-165.

[18] Mahl T, Hildebrandt A, Sawodny O J I T O R. A variable curvature continuum kinematics for kinematic control of the bionic handling assistant[J], 2014, 30(4): 935-949.

[19] Sears P, Dupont P. A steerable needle technology using curved concentric tubes[C]. $2006 \mathrm{IEEE} / \mathrm{RSJ}$ international conference on intelligent robots and systems, 2006: 2850-2856.

[20] Duleba I, Opalka M. A COMPARISON OF JACOBIAN-BASED METHODS OF INVERSE KINEMATICS FOR SERIAL ROBOT MANIPULATORS[J]. International Journal of Applied Mathematics and Computer Science, 2013, 23(2): 373-382.

[21] Jones B A, Walker I D. A new approach to Jacobian formulation for a class of multi-section continuum robots[C]. Proceedings of the 2005 IEEE International Conference on Robotics and Automation, 2005: 3268-3273

[22] Jiang H, Wang Z, Liu X, et al. A two-level approach for solving the inverse kinematics of an extensible soft arm considering viscoelastic behavior[C]. 2017 IEEE International Conference on Robotics and Automation (ICRA), 2017: 6127-6133.

[23] Melingui A, Merzouki R, Mbede J B, et al. Neural networks based approach for inverse kinematic modeling of a compact bionic handling assistant trunk[C]. 2014 IEEE 23rd International Symposium on Industrial Electronics (ISIE), 2014: 1239-1244.

[24] Thuruthel T G, Shih B, Laschi C, et al. Soft robot perception using embedded soft sensors and recurrent neural networks[J]. Science Robotics, 2019, 4(26).

[25] William Ii R L, Mayhew Iv J B. Obstacle-free control of the hyperredundant nasa inspection manipulator[C]. Proc. of the Fifth National Conf. on Applied Mechanics and Robotics, 1997: 12-15.

[26] Tappe S, Pohlmann J, Kotlarski J, et al. Towards a follow-the-leader control for a binary actuated hyper-redundant manipulator[C]. 2015 IEEE/RSJ International Conference on Intelligent Robots and Systems (IROS), 2015: 3195-3201.

[27] Neppalli S, Csencsits M A, Jones B A, et al. A Geometrical Approach to Inverse Kinematics for Continuum Manipulators[C]. IEEE/RSJ International Conference on Intelligent Robots and Systems, 2008: 3565-+.

[28] Gong Z, Fang X, Chen X, et al. A soft manipulator for efficient delicate grasping in shallow water: Modeling, control, and real-world experiments[J], 2021, 40(1): 449-469.

[29] Dong X, Raffles M, Cobos-Guzman S, et al. A Novel Continuum Robot Using Twin-Pivot Compliant Joints: Design, Modeling, and Validation[J]. Journal of Mechanisms and Robotics-Transactions of the Asme, 2016, 8(2).

[30] Yeshmukhametov A, Koganezawa K, Yamamoto Y, et al. Design and Kinematics of Cable-Driven Continuum Robot Arm with Universal Joint Backbone[C]. IEEE International Conference on Robotics and Biomimetics (ROBIO), 2018: 2444-2449.

\section{Biographical notes}

Haoran Wu, born in 1998, is currently a master candidate at Robotics Institute, Beihang University, China. His research interests include snake-arm robot and continuum robot.

E-mail: wuhaoran@buaa.edu.cn

Jingjun Yu, born in 1974, is currently a professor at Robotics Institute, Beihang University, China. He has published more than
160 papers. His research interests include mechanism and precision machinery.

E-mail: jjyu@buaa.edu.cn

Pan Jie, born in 1991, is currently a $\mathrm{PhD}$ candidate at Robotics Institute, Beihang University, China. His research interests include snake-arm robot and smart materials.

E-mail: panjie@buaa.edu.cn

Pei Xu, born in 1979, is currently an associate professor at Department of Mechanical Design, Beihang University, China. His research interests include mechanism and compliant mechanisms.

E-mail: peixu@buaa.edu.cn

\section{Appendix}

\section{Appendix A}

Table 2 Clustering algorithm to find the intersection of two curves

\begin{tabular}{|c|c|}
\hline \multicolumn{2}{|r|}{$\begin{array}{c}\text { Algorithm } 1 \text { Clustering algorithm to find the } \\
\text { intersection of two curves }\end{array}$} \\
\hline \multicolumn{2}{|c|}{ Input: $x, y, \theta$} \\
\hline \multicolumn{2}{|c|}{ Output: $P_{0}\left(x_{10}, y_{10}\right), P_{1}\left(x_{20}, y_{20}\right)$} \\
\hline & cal $S_{1}, S_{2}(\theta)$ \\
\hline & cal $P, x_{1-\max }, y_{1-\max }, x_{2-\max }, y_{2-\max }$ \\
\hline 3: & set $\begin{aligned} x_{10} & =x_{1-\min }+\left(x_{1-\max }-x_{1-\min }\right) \cdot \text { rand } \\
y_{10} & =y_{1-\min }+\left(y_{1-\max }-y_{1-\min }\right) \cdot \text { rand }\end{aligned}$ \\
\hline & set $\begin{aligned} x_{20} & =x_{2-\min }+\left(x_{2-\max }-x_{2-\min }\right) \cdot \text { rand } \\
y_{20} & =y_{2-\min }+\left(y_{2-\max }-y_{2-\min }\right) \cdot \text { rand }\end{aligned}$ \\
\hline 5: & set flag $=0$ \\
\hline & while flag $=0$ \\
\hline & while $\mathrm{i}<\mathrm{n}$ \\
\hline 8: & cal $\operatorname{dis}_{1}^{(i)}, \operatorname{dis}_{2}^{(i)}$ \\
\hline & if $\operatorname{dis}_{1}^{(i)}<\operatorname{dis}_{2}^{(i)}$ \\
\hline 10: & set indix $^{(i)}=1$ \\
\hline 11: & set. $\operatorname{var}_{1}=\operatorname{var}_{1}+d i s_{1}^{(i)}$. \\
\hline 12: & set num $_{1}=$ num $_{1}+1$ \\
\hline 13: & else \\
\hline $14:$ & set indix $^{(i)}=2$ \\
\hline 15: & set $\operatorname{var}_{2}=\operatorname{var}_{2}+d i s_{2}^{(i)}$ \\
\hline 16: & set num $_{2}=$ num $_{2}+1$ \\
\hline 17: & end if \\
\hline 18: & cal total $_{x-1}$, total $_{x-2}$, total $_{y-1}$, total $_{y-2}$ \\
\hline 19: & set $x_{10}=$ total $_{x-1} /$ num $_{1}, y_{10}=$ total $_{y-1} /$ num $_{1}$ \\
\hline 20: & set $x_{20}=$ total $_{x-2} /$ num $_{2}, y_{20}=$ total $_{y-2} /$ num $_{2}$ \\
\hline 21: & if $\operatorname{var}_{1}<\varepsilon \& \operatorname{var}_{2}<\varepsilon$ \\
\hline 22: & flag $=1$ \\
\hline 23: & end if \\
\hline 24: & end while \\
\hline
\end{tabular}


25: $\quad$ end while

26: return $P_{0}\left(x_{10}, y_{10}\right), P_{1}\left(x_{20}, y_{20}\right)$

\section{Appendix B}

Table 3 Solve the inverse kinematics of continuum robot

Algorithm 2 Solve the intersection of two curves in space

Input: $x, y, \theta$

Output: $\alpha, \beta$

1: $\quad$ cal $\theta_{\text {lef }}, \theta_{\text {right }}$ using Dichotomy

2: $\quad$ set $d \theta=\theta_{\text {right }}-\theta_{\text {left }}$

3: for $\theta=\theta_{\text {left }}: d \theta: \theta_{\text {right }}$

4: $\quad$ cal $S_{1}, S_{2}(\theta)$

5: $\quad$ cal $\left(x_{10}, y_{10}\right),\left(x_{20}, y_{20}\right)$ using Algorithm 1

6: $\quad$ cal $S_{1-2-a}, S_{1-2-b}, \operatorname{dis}\left(S_{1-2}\right)$

7: $\quad$ if $\operatorname{dis}\left(S_{1-2}\right)<\xi$

8: $\quad \quad \quad \quad$ al $\left(\alpha_{1}, \beta_{1}\right),\left(\alpha_{2}, \beta_{2}\right)$

9: $\quad$ end if

10: end for

11: $\quad$ return $(\alpha, \beta)$

\section{Appendix C}

$$
\begin{aligned}
& T=T_{1} \cdot T_{2}=\left[\begin{array}{cccc}
r 11 & r 12 & r 13 & r 14 \\
r 21 & r 22 & r 23 & r 24 \\
r 31 & r 32 & r 33 & r 34 \\
0 & 0 & 0 & 1
\end{array}\right] \\
& r_{11}=c_{\theta_{1}} c_{\theta_{2}}-c_{\alpha_{1}} s_{\theta_{1}} s_{\theta_{2}} \\
& r_{12}=c_{\alpha_{1}} c_{\alpha_{2}} c_{\theta_{2}} s_{\theta_{1}}-s_{\alpha_{1}} s_{\alpha_{2}} s_{\theta_{1}}+c_{\alpha_{2}} c_{\theta_{1}} s_{\theta_{2}} \\
& r_{13}=c_{\alpha_{2}} s_{\alpha_{1}} s_{\theta_{2}}+c_{\alpha_{1}} c_{\theta_{2}} s_{\alpha_{2}} s_{\theta_{2}}+c_{\theta_{1}} s_{\alpha_{2}} s_{\theta_{2}} \\
& r_{21}=-c_{\theta_{1}} s_{\theta_{2}}-c_{\alpha_{1}} c_{\theta_{1}} s_{\theta_{2}} \\
& r_{22}=c_{\alpha_{1}} c_{\alpha_{2}} c_{\theta_{1}} c_{\theta_{2}}-c_{\theta_{1}} s_{\alpha_{1}} s_{\alpha_{2}}-c_{\alpha_{2}} s_{\theta_{1}} s_{\theta_{2}} \\
& r_{23}=c_{\alpha_{2}} c_{\theta_{1}} s_{\alpha_{1}}+c_{\alpha_{1}} c_{\theta_{1}} c_{\theta_{2}} s_{\alpha_{2}}-s_{\alpha_{2}} s_{\theta_{1}} s_{\theta_{2}} \\
& r_{31}=s_{\alpha_{1}} s_{\theta_{2}} \\
& r_{32}=-c_{\alpha_{2}} c_{\theta_{2}} s_{\alpha_{1}}-c_{\alpha_{1}} s_{\alpha_{2}} \\
& r_{33}= c_{\alpha_{1}} c_{\alpha_{2}}-c_{\theta_{2}} s_{\alpha_{1}} s_{\alpha_{2}} \\
& r_{14}= \frac{l_{1} s_{\theta_{1}}\left(1-c_{\theta_{1}}\right)}{\alpha_{1}}+\frac{l_{2}\left(s_{\theta_{2}} c_{\theta_{1}}+c_{\theta_{2}} c_{\theta_{1}} s_{\alpha_{1}}\right)\left(1-c_{\alpha_{2}}\right)+l_{2} s_{\alpha_{2}} s_{\alpha_{1}} s_{\theta_{1}}}{\alpha_{2}} \\
& r_{24}= \frac{l_{1} c_{\theta_{1}}\left(1-c_{\theta_{1}}\right)}{\alpha_{1}}+\frac{l_{2}\left(s_{\theta_{2}} c_{\theta_{1}}+c_{\theta_{2}} c_{\theta_{1}} c_{\alpha_{1}}\right)\left(1-c_{\alpha_{2}}\right)+l_{2} s_{\alpha_{2}} s_{\alpha_{1}} c_{\theta_{1}}}{\alpha_{2}} \\
& r_{34}= \frac{l_{1} s_{\alpha_{1}}}{\alpha_{1}}+\frac{l_{2} s_{\alpha_{2}} c_{\alpha_{1}}-l_{2} c_{\theta_{2}} s_{\alpha_{1}}\left(1-c_{\alpha_{2}}\right)}{\alpha_{2}}
\end{aligned}
$$




\section{Supplementary Files}

This is a list of supplementary files associated with this preprint. Click to download.

- caltraji.xIsm

- resultja.xlsm

- resultmy.xlsm 